\title{
Over 15 years: the advancement of transcatheter aortic valve replacement
}

\author{
Thomas J. Cahill ${ }^{1}$, Juan A. Terre $^{2}$, Isaac George ${ }^{1}$ \\ ${ }^{1}$ Columbia University Irving Medical Center, NewYork-Presbyterian Hospital, New York, NY, USA; ${ }^{2}$ Montefiore Medical Center, Albert Einstein \\ College of Medicine, Bronx, NY, USA \\ Correspondence to: Isaac George, MD. 177 Fort Washington Avenue, 5th Floor, Room 5C-501, New York, NY 10032, USA. \\ Email: isaacgeorge@hotmail.com.
}

\begin{abstract}
The management of aortic stenosis has been revolutionized by transcatheter aortic valve replacement (TAVR). Initially only undertaken in patients at prohibitive or high surgical risk, as the evidence base and indications have expanded, TAVR is now approved and undertaken in patients at all risk levels. Evolution of valve technology, delivery systems and pathways for patient work-up have been rapid, with associated reductions in the complication profile, particularly vascular complications. Challenges remain as TAVR continues to advance, however, specifically achieving further reduction in paravalvular regurgitation, the requirement for permanent pacemaker implantation, and balancing the risks of thrombosis and bleeding. In this review, we outline the historical advances leading to contemporary TAVR practice, and discuss the future trajectory.
\end{abstract}

Keywords: Transcatheter aortic valve replacement (TAVR); surgical aortic valve replacement (SAVR); aortic stenosis; valvular heart disease

Submitted Sep 29, 2020. Accepted for publication Oct 21, 2020.

doi: 10.21037/acs-2020-av-24

View this article at: http://dx.doi.org/10.21037/acs-2020-av-24

\section{Introduction}

Over the last 15 years the management of aortic valve disease has been transformed by transcatheter aortic valve replacement (TAVR) (1). It has become the standard of care for patients with aortic stenosis (AS) at high surgical risk, and its use in intermediate- and low-risk groups is expanding rapidly, backed by randomized trial data across the spectrum of risk (2). TAVR continues to evolve rapidly, and improvements to the design of transcatheter heart valve (THV) and delivery systems, and the use of novel procedural adjuncts (e.g., for stroke protection, rhythm monitoring and vascular closure) are continuing to improve the predictability, safety and outcome of the procedure. In this review we summarize the history, recent procedural advances and future challenges for TAVR.

\section{History}

The invention of the heart-lung machine, first used in 1953, paved the way for the development of modern cardiac surgery and the first surgical aortic valve replacement (SAVR) using the Starr-Edwards prosthesis was carried out in 1961 (3). The initial steps toward minimally invasive management of AS came in 1985, with the first balloon aortic valvuloplasty (BAV) by Alain Cribier, in a 77-year-old woman with inoperable severe AS (4). While BAV produced an immediate improvement in valve hemodynamics, early valve restenosis affected as many as $80 \%$ by one year and its routine use was largely abandoned by the mid-1990s.

The key conceptual leap for TAVR was made by the Danish cardiologist Henning Rud Andersen, who conceived the idea of a crimped valve on a deflated balloon, allowing delivery through a low-profile sheath (5). He developed a porcine aortic valve sutured onto a metal stent, which could be inflated to expand the valve in the native aortic annulus, analogous to a coronary stent (Figure 1). Despite initial difficulty finding commercial support to transition into human studies, Percutaneous Valve Technologies was formed in 1999 with the aim of developing a balloonexpandable THV. 


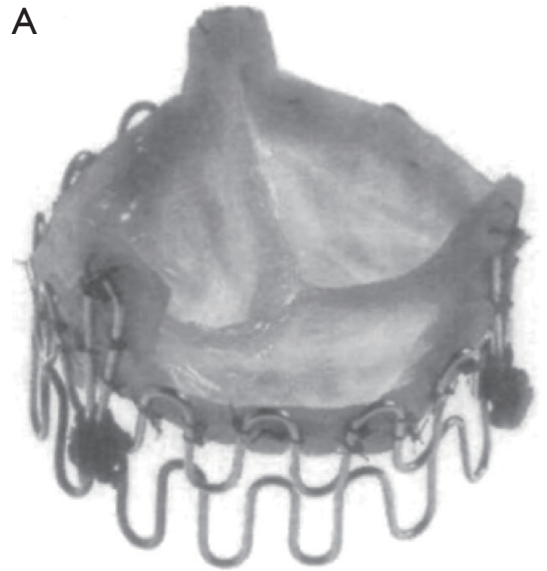

C

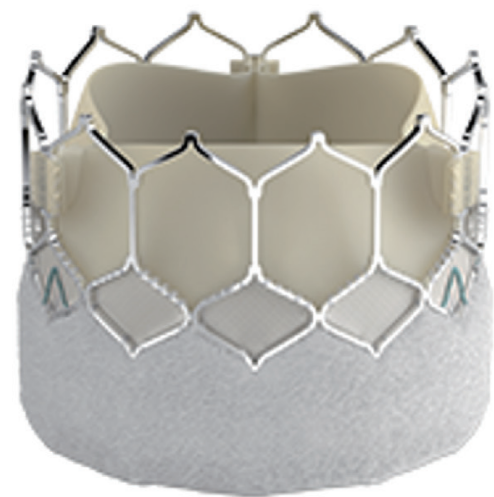

B

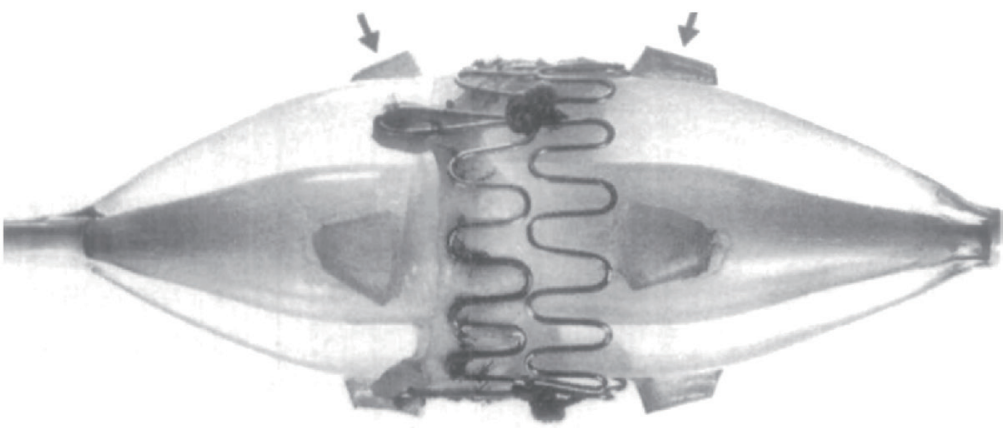

D

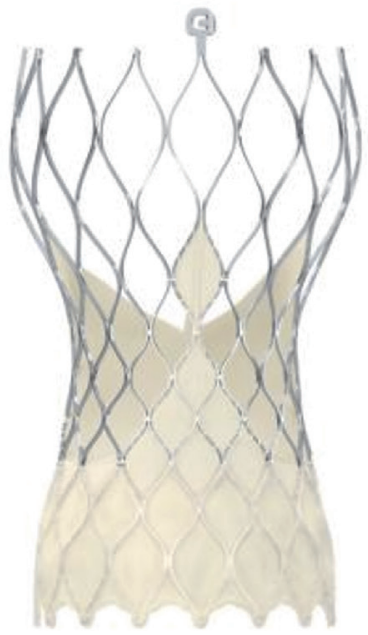

Figure 1 Transcatheter heart valves. The first transcatheter heart valve (THV) designed by Henning Rud Andersen, constructed by hand and implanted by catheter into the pig aorta (A,B). Contemporary design of a balloon-expandable valve (the SAPIEN 3 Ultra) (C) and selfexpandable valve (the Evolut Pro +) (D).

The first human TAVR was ultimately performed in Rouen in 2002 by Alain Cribier, in a 57-year-old man with critical AS and cardiogenic shock (6). The valve was delivered successfully via a transseptal approach and the patient survived to four months post-procedure before dying of unrelated complications. The success of this procedure lit the touch paper for TAVR: it confirmed the feasibility of transcatheter valve implantation in humans, with excellent hemodynamics, mild paravalvular aortic regurgitation and no embolization, coronary obstruction or atrioventricular (AV) block.

Transeptal TAVR proved reproducible and feasible in approximately $85 \%$ of cases, where it was conducted for compassionate use, but the takeover of Percutaneous Valve Technologies by Edwards Lifesciences in 2004 began the transition of TAVR into both a mainstream clinical and commercial reality (7). The early Cribier-Edwards 23-mm valve was redesigned and accompanied by delivery catheters for implantation of the valve from the retrograde transfemoral and antegrade transapical routes, opening the door for widespread adoption of the procedure.

\section{Clinical trials: from prohibitive to low risk patients}

The PARTNER series of randomized controlled trials have become the foundation of the evidence supporting TAVR. PARTNER B demonstrated the survival benefit of TAVR with the balloon-expandable SAPIEN THV (Edwards Lifesciences, CA, USA) over medical therapy in patients 
at prohibitive risk for SAVR, with a hazard ratio (HR) of 0.51 [95\% confidence interval (CI): 0.38-0.68, $\mathrm{P}<0.001$ ] for death over 24 months-one of the most impactful treatments seen in a contemporary randomised controlled trial (RCT) (8). PARTNER A, evaluating TAVR against SAVR in patients at high surgical risk, demonstrated noninferiority for TAVR for the composite primary endpoint of all-cause mortality or major stroke (HR 0.93, 95\% CI: 0.71-1.22, $\mathrm{P}=0.62$ ) (9). In the CoreValve US Pivotal study, the self-expanding CoreValve device (Medtronic, CA, USA) in patients at extreme risk for surgery was also associated with a significant reduction in mortality at 24 months (28.6\% vs. 22.2\%, $\mathrm{P}=0.04$ ) (10). These landmark studies drove an IA recommendation in the 2017 American College of Cardiology/The Society of Thoracic Surgeons (ACC/ STS) guidelines for TAVR for symptomatic patients with severe AS at prohibitive risk for SAVR, and the inclusion of TAVR as an alternative for those at high risk (11).

Two subsequent randomized trials evaluated the role of TAVR in patients at intermediate surgical risk: PARTNER $2 \mathrm{~A}$ and Surgical Replacement and Transcatheter Aortic Valve Implantation (SURTAVI). PARTNER 2A randomized 2,032 patients with a STS risk of $4-10 \%$ to TAVR with the SAPIEN XT valve, or SAVR (12). At two years, there was no significant difference in the primary endpoint, a composite of all-cause mortality or disabling stroke. SURTAVI enrolled 1,746 intermediate-risk patients (estimated surgical mortality 3-15\%), with randomization to TAVR using the self-expandable CoreValve system (CoreValve 84\%, Evolut R 16\%) or SAVR (13). SURTAVI also found no significant difference in the primary endpoint (the composite of death from any cause or disabling stroke) between TAVR and SAVR at a follow-up of two years. Based on non-inferiority of TAVR in these key trials, both European Guidelines and United States guidelines granted TAVR a recommendation for use in intermediate-risk patients, subject to a Heart Team evaluation of patient risk and anatomical profiles $(11,14)$.

Following on from the high- and intermediate-risk studies, PARTNER 3 and EVOLUT Low Risk evaluated TAVR against SAVR in patients at low surgical risk $(15,16)$. This group accounts for approximately $80 \%$ of patients currently undergoing SAVR (17). In PARTNER 3,1182 patients with an STS score of $<4 \%$ suitable for transfemoral TAVR (TF-TAVR) were randomized to TAVR or SAVR. At one year, the incidence of the primary endpoint of all-cause mortality, all stroke or cardiovascular rehospitalization was $15.1 \%$ for SAVR vs. $8.5 \%$ for TAVR,
HR 0.54 (95\% CI: $\left.0.37-0.79, \mathrm{P}_{\text {superiority }}=0.001\right)$ (15). For the key endpoint of death or disabling stroke, the incidence was significantly lower for TAVR compared with SAVR (2.9 vs. $1.0 \%$, HR 0.34, 95\% CI: 0.12-0.97, P=0.03). In the EVOLUT LR study, which used a Bayesian adaptive study design to evaluate TAVR with the self-expandable Evolut valve (Medtronic, Irvine, CA, USA), 1,468 patients were randomized to TAVR or SAVR. The composite endpoint of all-cause mortality or stroke at 24 months was $5.3 \%$ vs. $6.7 \%$, which was significant for non-inferiority (posterior probability of non-inferiority >0.999) (16).

In summary, both PARTNER 3 and Evolut LR demonstrated that TF-TAVR offers at least equivalence to SAVR in selected low-risk patients over a short followup period of one to two years. An updated meta-analysis of all randomized studies of TAVR against SAVR, across the spectrum of risk, demonstrated that TAVR is associated with a reduced risk of mortality (17\% relative risk reduction up to two years for TF-TAVR, when compared with SAVR; Figure 2). The risk of stroke, major bleeding, new-onset atrial fibrillation and acute kidney injury was also lower with TAVR than SAVR, but the risk of permanent pacemaker (PPM) implantation was higher (2). Importantly, however, low-risk patients with unfavourable anatomy (e.g., bicuspid valve, extensive left ventricular outflow tract calcification, poor transfemoral access) were excluded from the low-risk TAVR. The risk of paravalvular leak (PVL) remains higher with TAVR, and the durability of THVs remains uncertain. These issues represent significant ongoing challenges before TAVR will be ready for widespread use in the lowrisk population.

\section{Evolution of THV systems}

Since the first TAVR there have been huge engineering advances in both the design of THVs and the delivery system. The size of the access sheath required has decreased dramatically, from a 22-24 French (Fr) sheath for the original SAPIEN valve down to 14-16 Fr for the SAPIEN 3 (Figure $3 A$ ) and 14 Fr for the Evolut Pro Plus (Figure 3B). In turn, this has significantly reduced the number of vascular complications associated with the procedure, and increased the proportion of cases that can be carried out by the transfemoral approach. Valve design has been sequentially iterated over three generations of the balloon-expandable SAPIEN system (Edwards Lifesciences, CA, USA) and self-expandable CoreValve-Evolut system (Medtronic, CA, USA) to improve hemodynamics and function. In the 


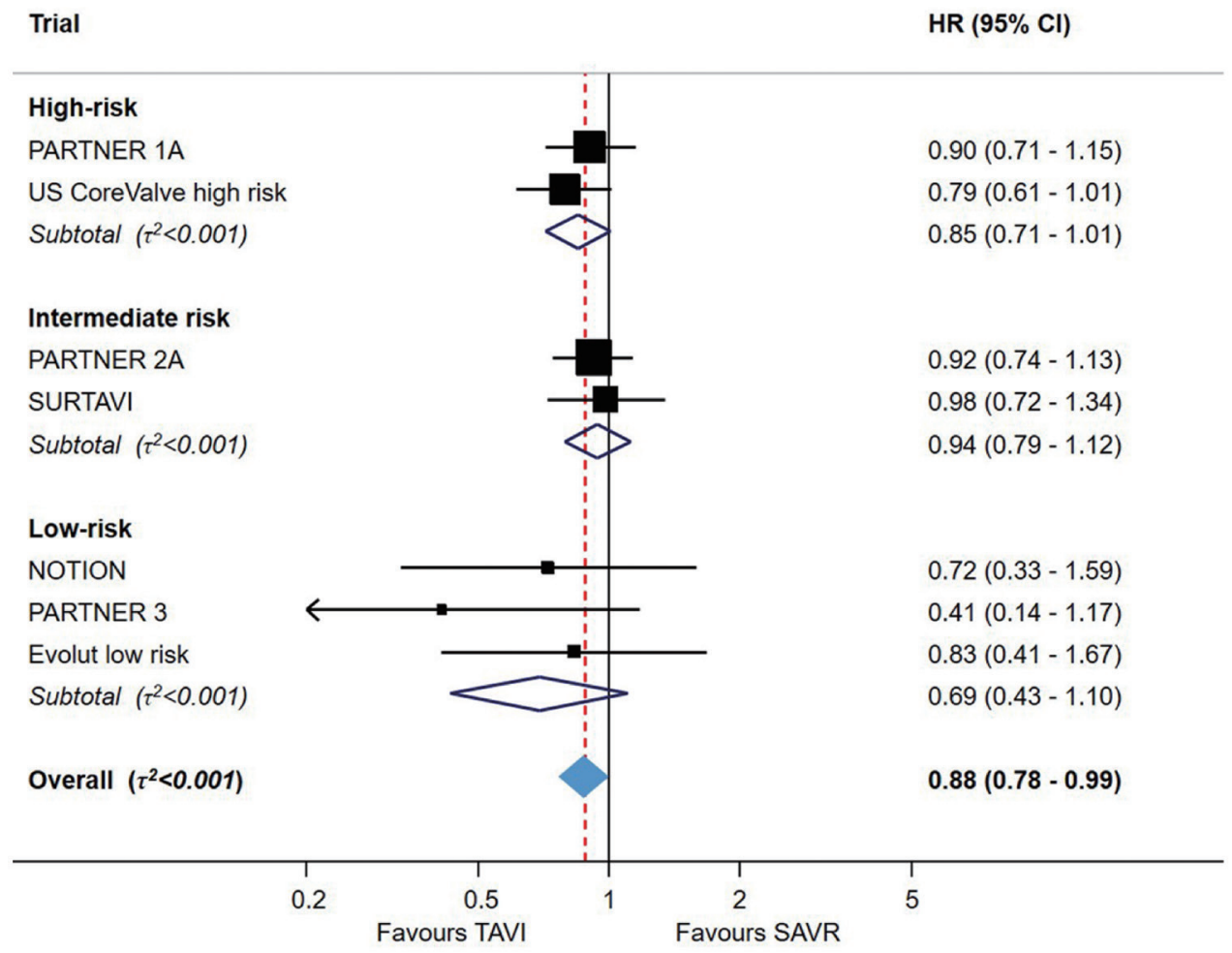

Figure 2 Mortality following TAVR $v s$. SAVR across the spectrum of risk. Meta-analysis of trials of transcatheter aortic valve implantation vs. surgical aortic valve replacement for mortality, up to 2-year follow-up. For each trial, boxes and horizontal lines correspond to the respective point estimate and accompanying $95 \%$ confidence interval. The size of each box is proportional to the weight of that trial result. The vertical solid line on the forest plot represents the point estimate of hazard ratio $=1$. The vertical dashed line on the plot represents the point estimate of overall hazard ratio derived from random-effect meta-analysis. The diamond represents the $95 \%$ confidence interval of the summary pooled estimate of the effect and is centred on pooled hazard ratios. Reproduced from Siontis et al. (2)

latest design of the SAPIEN 3, the Ultra, the height of the external polyethylene terephthalate (PET) skirt has been increased by $40 \%$ in an effort to reduce PVL. The Evolut PRO Plus range of valves has the broadest size range, from 23 to $34 \mathrm{~mm}$, and an external pericardial tissue wrap to improve sealing with the annulus. The mechanicallyexpandable Lotus Edge valve (Boston Scientific, Malborough, MA, USA), which is fully recapturable and repositionable with high radial strength, has been proposed to offer a theoretical advantage in very heavily calcified anatomy, but this is yet to be definitively demonstrated.

\section{Current challenges}

\section{Paravalvular leak}

PVL remains the Achilles' heel of TAVR, despite stepwise improvements as THV valve design has evolved. Predictive factors for PVL are heavy or asymmetric calcification, valve undersizing, device malpositioning, and incomplete apposition to the native aortic annulus $(18,19)$. SAVR has consistently outperformed TAVR for PVL in all trials to date. In PARTNER 2, at two years the incidence of greater/ equal to moderate PVL was $8.0 \%$ for TAVR arm, and $0.6 \%$ in the SAVR arm, with mild PVL seen in $28.6 \%$ after TAVR and $3.5 \%$ after SAVR (15). In PARTNER 3, although there was no difference in the incidence of greater/equal to moderate PVL at one year, the proportion of patients with mild PVL remained significantly higher $29.4 \%$ for TAVR, vs. $2.1 \%$ SAVR) (15).

The clinical impact of PVL is dependent on the severity of regurgitation. In patients receiving the SAPIEN 3 THV, greater/equal to moderate PVL at 30 days had a significantly higher one-year risk of all-cause mortality, with a HR of 2.59 (95\% CI: 1.39-4.85, $\mathrm{P}=0.003$ ), after adjustment 

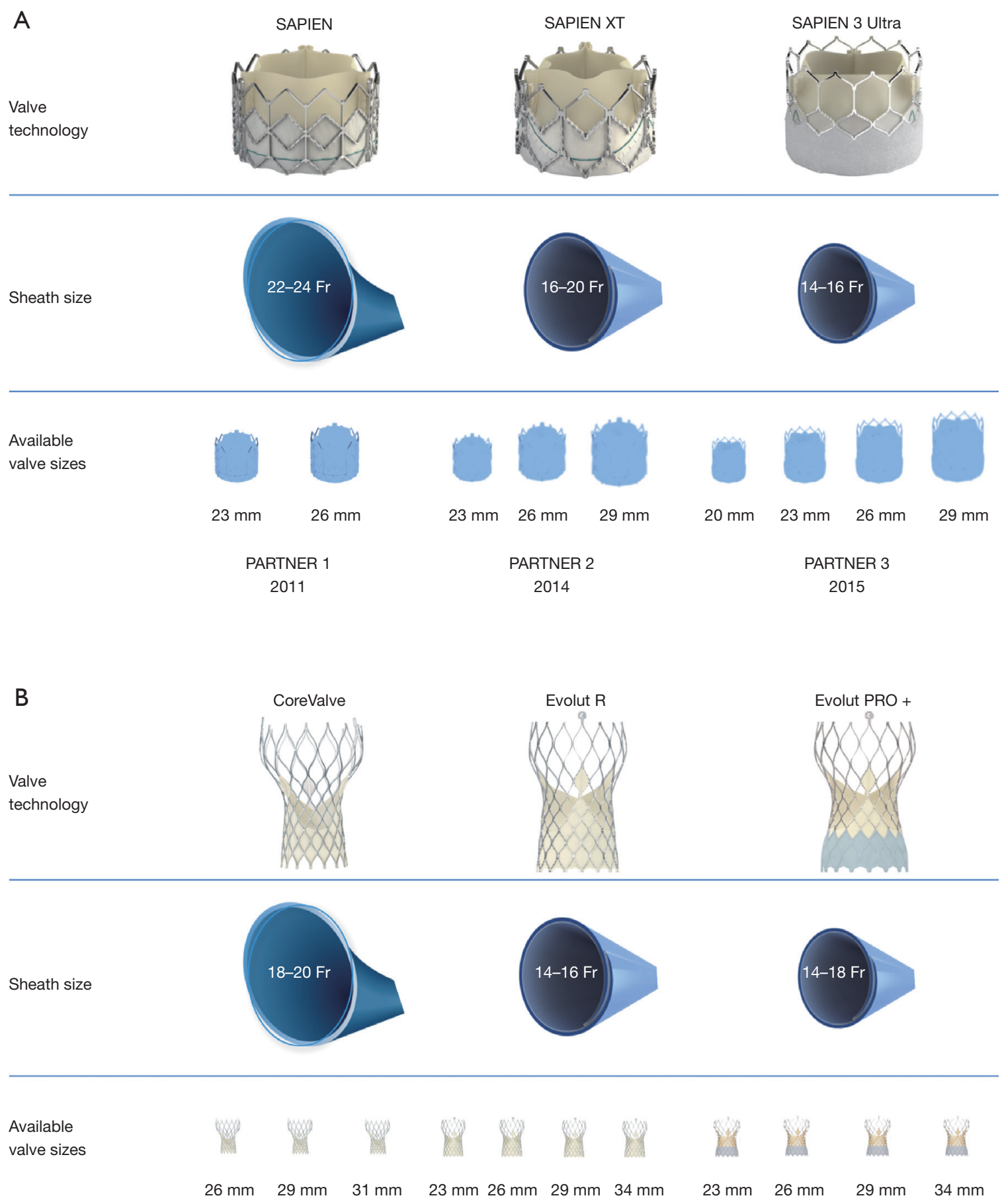

Figure 3 Evolution in transcatheter heart valves and delivery systems. The PARTNER randomized trials of TAVR $v s$. SAVR have implanted three generations of the SAPIEN transcatheter heart valve system. Over time, the valve system has been modified to increase the upper cell sizes (to facilitate coronary access), add an external skirt (to reduce paravalvular regurgitation), and the number of valve sizes available has increased. The latest generation of the SAPIEN 3, the Ultra, further increases the height of the external skirt. The sheath size for the delivery system has reduced from 22-24 Fr for the SAPIEN, to 14-16 Fr for the current SAPIEN 3. TAVR, transcatheter aortic valve replacement; SAVR, surgical aortic valve replacement. 
for baseline variables (20). Mild-moderate PVL has been linked to an increased risk of re-intervention. Ongoing debate exists around the significance of mild residual PVL and whether this may impact long-term outcomes, with conflicting study findings. In the era of minimalist TAVR, it can be challenging to identify the severity of PVL after valve deployment using fluoroscopy and transthoracic echocardiography alone and apply corrective interventions at the time. In some anatomies the severity of PVL can be reduced or abolished by valve post-dilatation. For balloonexpandable valves the valve delivery balloon is typically used, with up to 1-2 cc added to the balloon to appose the valve sealing skirt to the annulus. For self-expanding valves, balloon post-dilatation can be performed with sizing to the mean diameter of the annulus. For those with significant residual PVL not responsive to post-dilatation, options include targeted PVL closure or a subsequent valve-in-valve procedure.

\section{Stroke}

Stroke is often the most feared complication of TAVR. The majority of strokes are ischemic in etiology and occur in the first 24 hours of the procedure (21). Predictors include new-onset atrial fibrillation, prior stroke/transient ischemic attack, balloon post-dilatation, peripheral vascular disease and aortic atheroma (22). Major stroke after TAVR is associated with a devastating effect on survival, with average 30 -day mortality more than 3.5 -fold higher in patients with stroke (23).

Comparisons of stroke incidence between TAVR and SAVR have been heavily debated. In the high-risk populations studied in the PARTNER A and CoreValve High Risk trials, there was no statistically significant difference in the incidence of stroke between TAVR and SAVR, although TAVR had a numerical excess of strokes in both trials. In PARTNER 2, out to two-year followup, there was no significant difference in the incidence of disabling stroke or any stroke. Unexpectedly, in PARTNER 3 there were significantly more strokes seen in the SAVR arm, with an incidence of any stroke of $3.1 \%$ vs. $1.2 \%$ at 12 months (HR for TAVR 0.38, 95\% CI: 0.15-1.00, $\mathrm{P}=0.04)(15)$. The apparently high incidence of stroke after SAVR has been questioned, although it was similar in the Evolut LR trial, albeit with no clear difference to TAVR in that study (12-month incidence $4.1 \%$ TAVR vs. $4.3 \%$ SAVR, difference -0.2 , Bayesian credible interval for the difference, -2.4 to 1.9$)$ (16).

In addition to clinically detected transient ischemic attack and stroke, a significant proportion of patients undergoing TAVR sustain cerebral injury from embolic debris (24). The clinical consequences of this are not well established and while some events are entirely silent, cognitive decline, delirium and worsening memory function may result. The combination of clinical stroke with subclinical embolic events has led to the redoubling of efforts to develop devices which protect the brain. Despite the logical benefit of embolic protection devices, where filters are placed over the head and neck vessels to catch debris, providing evidence of benefit has been challenging. In the SENTINEL study, while there was a trend toward reduced infarct volume, this did not reach statistical significance and there was no difference in the rate of stroke (25). The REFLECTUS trial is an ongoing RCT evaluating the TriGuard device (Keystone Heart Ltd, Tampa, USA) in patients undergoing TAVR, which has now completed recruitment. Meta-analysis of studies of embolic protection suggest a benefit from their use (26), and a definitive trial of embolic protection showing reduction in clinical stroke would be a major advance for the TAVR field.

\section{Bleeding \& thrombosis}

Balancing the risks of bleeding and thrombosis, both for the periprocedural period and longer term, remains an ongoing challenge. On one side, major bleeding is an independent predictor of one-year mortality after TAVR, associated with an odds ratio (OR) of 2.36 (95\% CI: 1.68-3.31, P<0.0001) for death (27). On the other, TAVR valve implantation may carry higher risks of thrombosis than SAVR. Systematic computed tomography analysis of valve function has demonstrated subclinical high attenuation leaflet thickening (HALT) on $10-40 \%$ of THVs, in some cases associated with reduced leaflet motion and clinical sequelae of valve thrombosis (28). Thrombotic complications are potentially devastating to the patient's quality of life and also linked to an adverse outcome. Two key trials have pointed the way towards reducing routine use of anti-thrombotic and antiplatelet therapy after TAVR. First, the GALILEO trial compared Rivaroxaban with aspirin to dual antiplatelet therapy with aspirin and clopidogrel in patients without a conventional indication for anticoagulation (29). The trial was stopped prematurely by the data and safety monitoring board due to an excess of deaths in the Rivaroxaban group 
(5.8 vs. 3.4 per 100 person-years, HR 1.69, 95\% CI: $1.13-$ 2.53). Next, cohort B of the POPular TAVI trial, which compared the combination of oral anticoagulation (OAC) plus clopidogrel to OAC alone (30). POPular TAVI showed a lower incidence of bleeding in the OAC only arm, (risk ratio for OAC alone, $0.63 ; 95 \% \mathrm{CI}$ : $0.43-0.90 ; \mathrm{P}=0.01$ ) without an increased risk of thrombotic complications. Furthermore, in cohort A of the POPular TAVI study, amongst patients undergoing TAVI who did not have an indication for oral anticoagulation, the incidence of bleeding and the composite of bleeding or thromboembolic events at 1 year were significantly less frequent with aspirin alone compared with aspirin plus clopidogrel (31).

\section{Permanent pacemaker implantation}

Injury to the cardiac conduction system leading to PPM implantation remains an important limitation of TAVR. The incidence of pacemaker requirement after TAVR is influenced by THV choice, patient anatomy and implant technique. Although there are relatively few head-tohead comparisons between THVs, and so comparisons are primarily across studies, self-expanding and mechanically expandable valve systems are linked to higher rates of PPM. In the recent low-risk trials, the requirement for new PPM implantation was $7.3 \%$ for the SAPIEN 3 balloonexpandable valve, and $19.4 \%$ for the self-expanding Evolut valve. In the SOLVE-TAVI (Second-generation Selfexpandable Versus Balloon-expandable Valves and General Versus Local Anesthesia in TAVI) study, there was also a higher rate of pacing in patients receiving Evolut compared to the SAPIEN valve (32). By comparison, the incidence of PPM implantation after SAVR was $5.4 \%$ in PARTNER 3 and $6.7 \%$ in Evolut LR.

Evidence of conduction disease prior to TAVR, based on the pre-procedure electrocardiogram (ECG), is a strong predictor of a post-TAVR PPM. The strongest risk factor is the presence of right bundle branch block (RBBB), leading to an increased risk of PPM of at least three times and up to 47 times, and in some studies first degree AV block, left bundle branch block (LBBB) and left anterior hemiblock are associated with an increased risk of high-degree AV block, although the data are less consistent. In the PARTNER 2 cohort, RBBB was the strongest predictor of PPM implantation (OR 4.01, 95\% CI: 2.42-6.65, P<0.0001), followed by moderate-severe annular calcification (OR 2.04, 95\% CI: 1.29-3.23, $\mathrm{P}=0.0023$ ).
To an extent, the requirement for PPM is modifiable depending on procedural technique: low implantation depth, valve oversizing, larger valves and balloon valvuloplasty have all been linked to an increased PPM requirement. Low implant depth is associated with new LBBB and complete heart block, and reduction in implant depth across the learning curve for SAPIEN 3 was associated with reduced pacing rate (33). Implantation of a PPM is associated with adverse outcome-not only is there a procedural complication profile including infection, bleeding, hematoma, erosion and increased length of staybut PPM implantation is associated with an increased oneyear mortality (34). Furthermore, onset of new LBBB alone (in the absence of high degree AV block) is associated with increased all-cause mortality, cardiovascular mortality and rehospitalization $(35,36)$.

Novel approaches and valve designs are required to risk stratify, reduce injury to the conduction system and reduce the requirement for permanent pacing, particularly in younger patients. Risk models incorporating measurement of the membranous septum length in combination with ECG and assessment of calcium distribution may provide increased resolution for quantifying the risk of PPM (37). A modified implantation approach for self-expandable valves has recently been described, utilizing the "cusp overlap view", to facilitate higher implantation (38). In parallel, evolving pacing technology, such as the Micra leadless device, may in time reduce the morbidity with PPM implantation.

\section{Durability}

The durability of THVs remains a key unknown for TAVR, and is frequently highlighted as a barrier to extending the indication to younger patients.

The durability question has been limited by the lack of consistent criteria for defining structural valve deterioration (SVD), the relative importance placed on echocardiographic criteria, compared with symptoms or re-intervention, and a lack of side-by-side comparisons between TAVR and SAVR. Long-term follow-up after SAVR is available and whilst somewhat variable between valves and patient populations, has set the gold standard for durability. Over ten-year follow-up, freedom from reoperation was $97 \%$ and $99.6 \%$ for the Hancock II and Perimount valves respectively (Edwards Lifesciences, CA, USA) (39). For TAVR, medium-term follow-up over at least five years is 
now available for increasingly large populations. Toggweiler et al. (40) reported promising five-year outcomes from the first generation Sapien valve. At PCR London Valves 2019, five-year follow-up of PARTNER 2A suggested significantly higher rate of SVD in recipients of the SAPIEN XT valve compared with SAVR (8.8\% vs. 3.5\%, HR 0.41, 95\% CI: $0.23-0.74, \mathrm{P}=0.002$ ), although the SAPIEN 3 valve was no different to surgery (41). Given the continued evolution of THV devices, with successive iterations prior to the availability of long-term (ten-year) durability data, it is likely that significant transition will occur into younger patients before there is a clear answer regarding valve durability.

\section{Conclusions}

The combination of unmet clinical need, engineering breakthroughs and construction of a rigorous evidence base has allowed TAVR to transform the management of AS over the last 15 years. Whilst challenges remain, TAVR is continuing to grow rapidly and ultimately will become the treatment of choice for the majority of patients with AS. TAVR is now increasingly recognized as an appropriate treatment modality for patients with suitable anatomy across the spectrum of risk. In rapidly changing landscape there are few certainties, but today and over the next 15 years, the role of the heart valve team comprising cardiologists and surgeons working side-by-side, looks set to remain the foundation of delivering care to patients with heart valve disease.

\section{Acknowledgments}

Funding: None.

\section{Footnote}

Conflicts of Interest: The authors have no conflicts of interest to declare.

Open Access Statement: This is an Open Access article distributed in accordance with the Creative Commons Attribution-NonCommercial-NoDerivs 4.0 International License (CC BY-NC-ND 4.0), which permits the noncommercial replication and distribution of the article with the strict proviso that no changes or edits are made and the original work is properly cited (including links to both the formal publication through the relevant DOI and the license). See: https://creativecommons.org/licenses/by-nc-nd/4.0/.

\section{References}

1. Cahill TJ, Chen M, Hayashida K, et al. Transcatheter aortic valve implantation: current status and future perspectives. Eur Heart J 2018;39:2625-34.

2. Siontis GCM, Overtchouk P, Cahill TJ, et al. Transcatheter aortic valve implantation vs. surgical aortic valve replacement for treatment of symptomatic severe aortic stenosis: an updated meta-analysis. Eur Heart J 2019;40:3143-53.

3. Effler DB, Favaloro R, Groves LK. Heart Valve Replacement: Clinical Experience. Ann Thorac Surg 1965;1:4-24.

4. Cribier A, Savin T, Saoudi N, et al. Percutaneous transluminal valvuloplasty of acquired aortic stenosis in elderly patients: an alternative to valve replacement? Lancet 1986;1:63-7.

5. Andersen HR, Knudsen LL, Hasenkam JM. Transluminal implantation of artificial heart valves. Description of a new expandable aortic valve and initial results with implantation by catheter technique in closed chest pigs. Eur Heart J 1992;13:704-8.

6. Cribier A, Eltchaninoff H, Bash A, et al. Percutaneous Transcatheter Implantation of an Aortic Valve Prosthesis for Calcific Aortic Stenosis: First Human Case Description. Circulation 2002;106:3006-8.

7. Cribier A, Eltchaninoff H, Tron C, et al. Treatment of Calcific Aortic Stenosis With the Percutaneous Heart Valve: Mid-Term Follow-Up From the Initial Feasibility Studies: The French Experience. J Am Coll Cardiol 2006; 47:1214-23.

8. Leon MB, Smith CR, Mack M, et al. Transcatheter Aortic-Valve Implantation for Aortic Stenosis in Patients Who Cannot Undergo Surgery. N Engl J Med 2010;363:1597-607.

9. Smith CR, Leon MB, Mack MJ, et al. Transcatheter versus Surgical Aortic-Valve Replacement in High-Risk Patients. N Engl J Med 2011;364:2187-98.

10. Popma JJ, Adams DH, Reardon MJ, et al. Transcatheter Aortic Valve Replacement Using a Self-Expanding Bioprosthesis in Patients with Severe Aortic Stenosis at Extreme Risk for Surgery. J Am Coll Cardiol 2014;63:1972-81.

11. Nishimura RA, Otto CM, Bonow RO, et al. 2017 AHA/ 
ACC Focused Update of the 2014 AHA/ACC Guideline for the Management of Patients With Valvular Heart Disease: A Report of the American College of Cardiology/ American Heart Association Task Force on Clinical Practice Guidelines. Circulation 2017;135:e1159-95.

12. Leon MB, Smith CR, Mack MJ, et al. Transcatheter or Surgical Aortic-Valve Replacement in Intermediate-Risk Patients. N Engl J Med 2016;374:1609-20.

13. Reardon MJ, Van Mieghem NM, Popma JJ, et al. Surgical or Transcatheter Aortic-Valve Replacement in Intermediate-Risk Patients. N Engl J Med 2017;376:1321-31.

14. Baumgartner H, Falk V, Bax JJ, et al. 2017 ESC/EACTS Guidelines for the management of valvular heart disease. Eur Heart J 2017;38:2739-91.

15. Mack MJ, Leon MB, Thourani VH, et al. Transcatheter Aortic-Valve Replacement with a Balloon-Expandable Valve in Low-Risk Patients. N Engl J Med 2019;380:1695-705.

16. Popma JJ, Deeb GM, Yakubov SJ, et al. Transcatheter Aortic-Valve Replacement with a Self-Expanding Valve in Low-Risk Patients. N Engl J Med 2019;380:1706-15.

17. Thourani VH, Suri RM, Gunter RL, et al. Contemporary Real-World Outcomes of Surgical Aortic Valve Replacement in 141,905 Low-Risk, Intermediate-Risk, and High-Risk Patients. Ann Thorac Surg 2015;99:55-61.

18. Athappan G, Patvardhan E, Tuzcu EM, et al. Incidence, Predictors, and Outcomes of Aortic Regurgitation After Transcatheter Aortic Valve Replacement: Meta-Analysis and Systematic Review of Literature. J Am Coll Cardiol 2013;61:1585-95.

19. Khalique OK, Hahn RT, Gada H, et al. Quantity and Location of Aortic Valve Complex Calcification Predicts Severity and Location of Paravalvular Regurgitation and Frequency of Post-Dilation After Balloon-Expandable Transcatheter Aortic Valve Replacement. JACC Cardiovasc Interv 2014;7:885-94.

20. Pibarot P, Hahn RT, Weissman NJ, et al. Association of Paravalvular Regurgitation With 1-Year Outcomes After Transcatheter Aortic Valve Replacement With the SAPIEN 3 Valve. JAMA Cardiol 2017;2:1208-16.

21. Kapadia S, Agarwal S, Miller DC, et al. Insights Into Timing, Risk Factors, and Outcomes of Stroke and Transient Ischemic Attack After Transcatheter Aortic Valve Replacement in the PARTNER Trial (Placement of Aortic Transcatheter Valves). Circ Cardiovasc Interv 2016;9:e002981.
22. Auffret V, Regueiro A, Del Trigo M, et al. Predictors of Early Cerebrovascular Events in Patients With Aortic Stenosis Undergoing Transcatheter Aortic Valve Replacement. J Am Coll Cardiol 2016;68:673-84.

23. Eggebrecht H, Schmermund A, Voigtländer T, et al. Risk of stroke after transcatheter aortic valve implantation (TAVI): a meta-analysis of 10,037 published patients. EuroIntervention 2012;8:129-38.

24. Van Mieghem NM, El Faquir N, Rahhab Z, et al. Incidence and Predictors of Debris Embolizing to the Brain During Transcatheter Aortic Valve Implantation. JACC Cardiovasc Interv 2015;8:718-24.

25. Kapadia SR, Kodali S, Makkar R, et al. Protection Against Cerebral Embolism During Transcatheter Aortic Valve Replacement. J Am Coll Cardiol 2017;69:367-77.

26. Giustino G, Sorrentino S, Mehran R, et al. Cerebral Embolic Protection During TAVR: A Clinical Event Meta-Analysis. J Am Coll Cardiol 2017;69:465-6.

27. Généreux P, Cohen DJ, Williams MR, et al. Bleeding Complications After Surgical Aortic Valve Replacement Compared With Transcatheter Aortic Valve Replacement: Insights From the PARTNER I Trial (Placement of Aortic Transcatheter Valve). J Am Coll Cardiol 2014;63:1100-9.

28. Makkar RR, Fontana G, Jilaihawi H, et al. Possible Subclinical Leaflet Thrombosis in Bioprosthetic Aortic Valves. N Engl J Med 2015;373:2015-24.

29. Dangas GD, Tijssen JGP, Wöhrle J, et al. A Controlled Trial of Rivaroxaban after Transcatheter Aortic-Valve Replacement. N Engl J Med 2020;382:120-9.

30. Nijenhuis VJ, Brouwer J, Delewi R, et al. Anticoagulation with or without Clopidogrel after Transcatheter AorticValve Implantation. N Engl J Med 2020;382:1696-707.

31. Brouwer J, Nijenhuis VJ, Delewi R, et al. Aspirin with or without Clopidogrel after Transcatheter Aortic-Valve Implantation. N Engl J Med 2020;383:1447-57.

32. Thiele H, Kurz T, Feistritzer HJ, et al. Comparison of newer generation self-expandable vs. balloon-expandable valves in transcatheter aortic valve implantation: the randomized SOLVE-TAVI trial. Eur Heart J 2020;41:1890-9.

33. Binder RK, Webb JG, Toggweiler S, et al. Impact of Post-Implant SAPIEN XT Geometry and Position on Conduction Disturbances, Hemodynamic Performance, and Paravalvular Regurgitation. JACC Cardiovasc Interv 2013;6:462.

34. Nazif TM, Dizon José M, Hahn RT, et al. Predictors and Clinical Outcomes of Permanent Pacemaker Implantation 
After Transcatheter Aortic Valve Replacement: The PARTNER (Placement of AoRtic TraNscathetER Valves) Trial and Registry. JACC Cardiovasc Interv 2015;8:60-9.

35. Nazif TM, Chen S, George I, et al. New-onset left bundle branch block after transcatheter aortic valve replacement is associated with adverse long-term clinical outcomes in intermediate-risk patients: an analysis from the PARTNER II trial. Eur Heart J 2019;40:2218-27.

36. Faroux L, Chen S, Muntané-Carol G, et al. Clinical impact of conduction disturbances in transcatheter aortic valve replacement recipients: a systematic review and meta-analysis. Eur Heart J 2020;41:2771-81.

37. Maeno Y, Abramowitz Y, Kawamori H, et al. A Highly Predictive Risk Model for Pacemaker Implantation After

Cite this article as: Cahill TJ, Terre JA, George I. Over 15 years: the advancement of transcatheter aortic valve replacement. Ann Cardiothorac Surg 2020;9(6):442-451. doi: 10.21037/acs-2020-av-24
TAVR. JACC Cardiovasc Imaging 2017;10:1139-47.

38. Tang GHL, Zaid S, Michev I, et al. "Cusp-Overlap" View Simplifies Fluoroscopy-Guided Implantation of Self-Expanding Valve in Transcatheter Aortic Valve Replacement. JACC Cardiovasc Interv 2018;11:1663-5.

39. Rahimtoola SH. Choice of Prosthetic Heart Valve in Adults: An Update. J Am Coll Cardiol 2010;55:2413-26.

40. Toggweiler S, Humphries KH, Lee M, et al. 5-year outcome after transcatheter aortic valve implantation. J Am Coll Cardiol 2013;61:413-9.

41. Pibarot P, Ternacle J, Jaber WA, et al. Structural Deterioration of Transcatheter Versus Surgical Aortic Valve Bioprostheses in the PARTNER-2 Trial. J Am Coll Cardiol 2020;76:1830-43. 